\title{
Polyclonal T Lymphocytes Transform into Malignant Cells by Retroviral Mediated Transfer of p21SNFT Gene
}

\author{
Ashok Kumar ${ }^{1, *}$, Bharathi Sengodan ${ }^{1}$, Chetan Lal ${ }^{2}$, Anthony Leela ${ }^{3}$, Kevin Fernandez ${ }^{3}$ \\ 'Department of Pathology, Faculty of Medicine, AIMST University, MALAYSIA. \\ 2Department of Microbiology, Federal Postgraduate Medical Institute, Lahore, Punjab, PAKISTAN. \\ 3Department of Community Medicine, Faculty of Medicine, AIMST University, MALAYSIA.
}

\begin{abstract}
Background: p21SNFT (21 kDa small nuclear factor isolated from T cells) gene is known to be increasingly expressed in the cells of Hodgkin disease and Hodgkin cell lines. It was not investigated before if this gene had any effect on polyclonal T lymphocytes. For this purpose, we transduced this gene into polyclonal T lymphocytes and transplanted into mice. Materials and Methods: We used $\boldsymbol{y}$-retroviral vector to transduce this gene into polyclonal T lymphocytes, isolated from wild type black-6 mice. After confirmation of p21SNFT expression via marker gene eGFP (enhanced green fluorescent protein) and Western blot analysis, these cells were transplaned into immunocompromised Rag 1 deficient mice. The control group of mice was transplanted with $\gamma$-retroviral vector, carrying only marker gene (eGFP). The mice were followed up. The results showed that all 7 mice transplanted with p21SNFT-carrying polyclonal T lymphocytes developed massively enlarged mesenteric lymph nodes after 435 to 550 days of transplantation. Results: Histopathological analyses of these lymph nodes revealed the lymphoma, which resembled human peripheral T cell lymphoma
\end{abstract}

type. Furthermore, immunophenotyping of tumor cells by FACS analysis revealed loss of $\mathrm{T}$ cell markers and expression of CD19 marker. From the control group, all five mice remained healthy and did not develop any tumor. Conclusion: The study concludes that $\gamma$-retroviral mediated transfer of p21SNFT transforms polyclonal T lymphocytes into malignant cells.

Key words: $\gamma$-retroviral vector, Lymphoma, Malignant transformation, p21SNFT, T lymphocytes.

Correspondence

Dr. Ashok Kumar (MBBS, DCP, PhD, Postdoc fellowship)

Associate Professor, Department of Pathology, Aimst University, Bedong, Kedah-08100 Semeling, MALAYSIA.

Phone: +60146895786

Email: dr.ashok.kumar@hotmail.com

DOI: 10.5530/jyp.2020.12.14

\section{INTRODUCTION}

The gene p21SNFT (21 kDa small nuclear factor isolated from T cells) encodes a basic leucine zipper protein. ${ }^{1}$ Through Serial analysis of gene expression and semi-quantitative RT/PCR analyses, this gene was found to be up regulated in Hodgkin disease (HD) and Hodgkin cell lines. ${ }^{2,3}$ Since this gene was upregulated in Hodgkin disease cells, we wanted to further investigate if $p 21$ SNFT could have oncogenic potential for lymphocytes. In the current study we selected wild type polyclonal T lymphocytes. T lymphocytes, which are produced by hematopoietic stem cells in the bone marrow, migrate to the thymus for further processing of selection and maturation. Afterwards, they leave thymus and enter the blood circulation and lymphatic system. ${ }^{4,5} \mathrm{~T}$ lymphocytes get their stimulatory niche through their receptors TCRs (T cell receptors), by interacting with molecule spMHC (self-peptide major histocompatibility complex) expressed on antigen presenting cells. In a human or a wild type mouse, these lymphocytes express different types of TCRs, hence are polyclonal. This polyclonality provides the lymphocytes with a variety of TCR expressing T lymphocytes, which interact and fight a variety of pathogens. Thus, T lymphocyte diversity provides us with a powerful defense system against a variety of pathogens. ${ }^{6,7}$ In the current experimental setup, we chose T lymphocytes for two reasons. First, these cells have been used in gene therapy. ${ }^{8}$ Therefore, we wanted to further confirm the safety issue of these cells in gene therapy. Second, as p21SNFT gene was found to be upregulated in Hodgkin disease cells and Hodgkin disease has been investigated to originate from $\mathrm{B}^{9}$ and $\mathrm{T}$ lymphocytes. ${ }^{10}$ Hence, we wanted to investigate its oncogenic potential in polyclonal $\mathrm{T}$ lymphocytes.
We used Rag 1 deficient mice as the recipients of $p 21 S N F T$ transduced $T$ lymphocytes. These mice are immunodeficient as they do not have mature $\mathrm{T}$ and $\mathrm{B}$ lymphocytes, hence easily accept the transplanted cells as there would be no immune rejection.

For the transfer of p21SNFT gene into polyclonal T lymphocytes, we used $\gamma$-retroviral vector. This vector is derived from enveloped RNA virus. The vector carries the desired gene into the target cell where it reverse-transcribes to DNA and integrates into the host genome. These vectors are already reported to support a long term expression of the transgene. ${ }^{11}$ Therefore, we used $\gamma$-retroviral vector to transfer $p 21 S N F T$ gene. Hence, by using this setup, we could transduce $p 21 S N F T$ effectively with its full expression on protein level in polyclonal $\mathrm{T}$ lymphocytes and investigate its effects.

\section{MATERIAL AND METHODS}

\section{Gammaretroviral vector and packaging of p21snft gene}

A $\gamma$-retroviral vector MP91/eGFP was used to transfer $p 21 S N F T$ gene into polyclonal T lymphocytes. Empty MP91/eGFP vector was used as a control as described. ${ }^{12}$ We purchased the cDNA of human $p 21 S N F T$ gene from GeneArt Company and cloned it into MP91/eGFP vector. This vector codes for eGFP (enhanced green fluorescent protein), which acts as a marker gene as it fluoresces after it is expressed in the cells. The gene p21SNFT was cloned in MP91/eGFP vector as described. ${ }^{12}$

Figure 1 shows the control $\gamma$-retroviral vector 'MP91/eGFP' and p21SNFT-cloned $\gamma$-retroviral vector 'MP91/p21SNFT'.

This is an open access article distributed under the terms of the Creative Commons Attribution-NonCommercial-ShareAlike 4.0 License, which allows others to remix, tweak, and build upon the work non-commercially, as long as the author is credited and the new creations are licensed under the identical terms. 


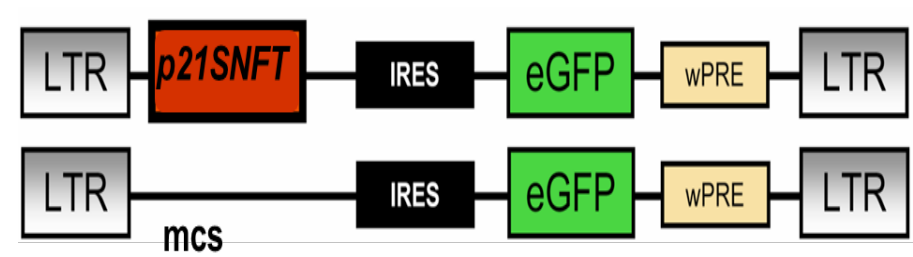

Figure 1: Molecular map of $\mathrm{\gamma}$-retroviral vector. MP91/p21SNFT vector (upper). Control vector MP91/eGFP (lower). p21SNFT gene was cloned into multiple cloning site (mcs) of the control vector. IRES: Internal ribosomal entry site; eGFP: Enhanced green fluorescent protein; wPRE: Woodchuck hepatitis virus posttranscriptional regulatory element; LTR: Long terminal repeat.

The MP91/eGFP and MP91/p21SNFT vectors were packaged by calcium phosphate mediated transient transfection of 293 T cells as described. ${ }^{12}$ The supernatant, containing the viral particles (eGFP / p21SNFT) were filtered through the strainer and used to transduce polyclonal $\mathrm{T}$ lymphocytes.

\section{Cell culture and animal experimental work}

Animal experiments were approved by the Regional Council Darmstadt. For animal experiments, mice were purchased from Charles River Laboratories (Sulzfeld, Germany) and The Jackson Laboratory (Bar Harbor, USA).

Polyclonal T lymphocytes were isolated from six to eight weeks old wild type black 6 mice. These donor mice were first anesthetized with isofluran and then sacrificed by dislocating the cervical spines. $70 \%$ ethanol was sprayed over the skin to disinfect the abdominal and chest parts of the sacrificed mice. After careful dissection, spleen and mesenteric lymph nodes were excised and homogenized with $1 \%$ PBS buffer. The cells were filtered through cell strainer and washed with 1\% PBS buffer. Isolated cells were stimulated with anti CD3/CD28 antibodies-coupled paramagnetic beads for four days. The cells were analyzed by using FACS (fluorescent activated cell sorting) to confirm the expression of $\mathrm{T}$ cell markers CD 3 and TCR $\alpha / \beta$.

Afterwards, these polyclonal $\mathrm{T}$ lymphocytes were transduced with the MP91/p21SNFT and control (MP91/eGFP) supernatants. The transgene expression, represented by eGFP expression, was measured after $36 \mathrm{hr}$ by FACS. Further confirmation of p21SNFT gene on protein level expression was done by performing Western blot. The cells were then washed with $1 \%$ PBS buffer and transplanted into RAG 1 deficient recipient mice. $1-2 \times 10^{7}$ cells / mouse were injected into tail vein. 7 mice were transplanted with p21SNFT transduced polyclonal T lymphocytes and 5 mice were transplanted with the eGFP transduced polyclonal T lymphocytes which served as the control group. After follow up of $\sim 12$ months, the mice were sacrificed due to limited life expectancy and the cells were isolated from spleen and mesenteric lymph nodes. After homogenizing and washing with $1 \%$ PBS buffer and confirming by FACS analysis for eGFP expression, the cells were serially transplanted into 6 to 8 weeks old RAG 1 deficient mice. The protocol was followed as described. ${ }^{12,13}$

\section{Blood analysis}

Blood was withdrawn from lateral tail vein by making a small incision with a scalpel during the experimental time, or directly by puncturing the heart after sacrificing the mice. 50-100 $\mu \mathrm{l}$ of blood was collected in an EDTA coated capillary (Microvette) for FACS and hemogram analyses. Hemogram was performed using Scil vet animal blood counter (Scil Animal Care, Viernheim, Germany).

\section{Histopathological analysis}

Mice with the signs of sickness, or enlarged abdomen were sacrificed by cervical dislocation. Spleen, liver, lymph nodes and bone marrow were isolated and fixed in Zinc Formal Fixx (Thermo Shandon, Pittsburgh, USA). After 24 hours, the Zinc Formal Fixx was exchanged with 70\% ethanol. Histological sections and diagnoses were done by Pathology Institute at University of Frankfurt.

\section{FACS analysis}

$\sim 5 \times 10^{5}$ cells from each sample (blood / tumor mass) were used. Each sample was measured in triplicate. The tumor mass was homogenized with the stamp of a syringe through a cell strainer in a 6-well plate and put in a $5 \mathrm{ml}$ polystyrol FACS tube. The cells were washed with $1 \times \mathrm{PBS}$ buffer and suspended in $200 \mu \mathrm{l}$ PBS. $1 \mu \mathrm{l}$ of each anti-mouse antibody for CD3, TCR $\alpha / \beta$, CD19, CD45.1 and CD45.2 markers (Invitrogen) were added. After incubating the cells for $15 \mathrm{~min}$. in dark, Cal-lysis solution (Invitrogen) was added to the samples to lyse red blood cells (RBCs). The cells were washed three times in $3 \mathrm{ml}$ of $1 \times$ PBS buffer by centrifuging at $1400 \mathrm{rpm} / 3 \mathrm{~min}$. The supernatant was discarded and the cells were resuspended in $400 \mu \mathrm{l}$ of $1 \times$ PBS buffer. The samples were analyzed using FACSCalibur and Cellquest pro software.

\section{Western blotting}

Western blotting was performed for the confirmation of expression of p21SNFT on protein level. $3 \times 10^{6}$ tumor cells were washed three times with $3 \mathrm{ml}$ cold sterile $1 \mathrm{x}$ PBS buffer by centrifuging for $2 \mathrm{~min}$ at 13000 $\mathrm{rpm}$. The cell pellets were resuspended in ice-cold lysis buffer and vortexed and incubated for $15 \mathrm{~min}$. on ice. The lysates were then spinned down for $10 \mathrm{~min}$. at $13000 \mathrm{rpm}$ in a microcentrifuge $\left(4^{\circ} \mathrm{C}\right)$ to settle the cell debris down. Proteins in the supernatant were harvested and transferred to a $1.5 \mathrm{ml}$ reaction tube. Mouse anti p21SNFT antibodies (Abnova) were used for blotting. The detailed protocol was followed as described. ${ }^{12}$

\section{RESULTS}

All 7 mice, transplanted with $p 21 S N F T$-transduced polyclonal T lymphocytes developed lymphoma after 435 to 550 days of transplantation. Figure 2 shows the survival curve.

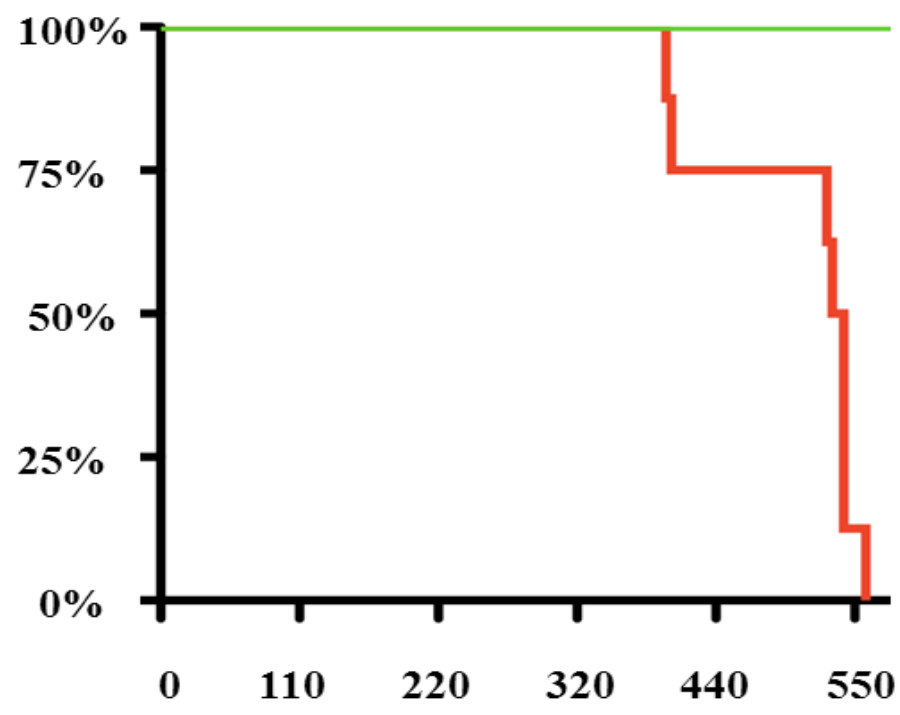

Figure 2: Survival curve of mice. All mice from p21SNFT group developed lymphoma (red line) after 435 to 550 days of transplantation. None of the mice from control group (green line) developed any malignancy. 
The mice developed enlarged belly and loss of weight. Mice were sacrificed and immediately blood samples were collected from the heart with the help of a syringe. Blood complete pictures were performed, which did not show any abnormality. For further examination, autopsies of the mice were performed, which showed massively enlarged mesenteric lymph nodes (Figure 3a). The histopathological analyses of these masses showed diffusely spread intermediate sized mature lymphocytes with clear cytoplasm and oval nucleus, comprising the features of peripheral T cell lymphoma (Figure 3b).

For further investigation, FACS analysis was performed to determine the phenotype of the tumor cells. All the tumors showed similar phenotypes. All eGFP expressing cells were positive for CD19 and negative for CD3 and TCR $\alpha / \beta$ (Figure 4 ). For further confirmation, Western blotting was performed, which showed the expression of p21SNFT on protein level in tumor cells (Figure 5).

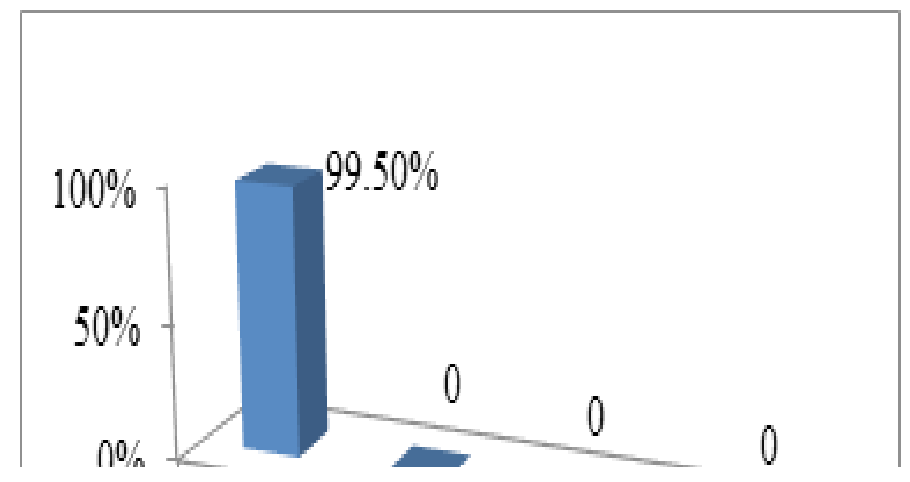

Figure 3: (a) Gross features of tumor mass. Grossly enlarged mesenteric lymph node. (b) Histopathological features of enlarged lymph node. Diffusely spread intermediate sized mature lymphocytes with clear cytoplasm and oval nucleus, comprising the features of peripheral T cell lymphoma (H\&E staining, magnification X40).

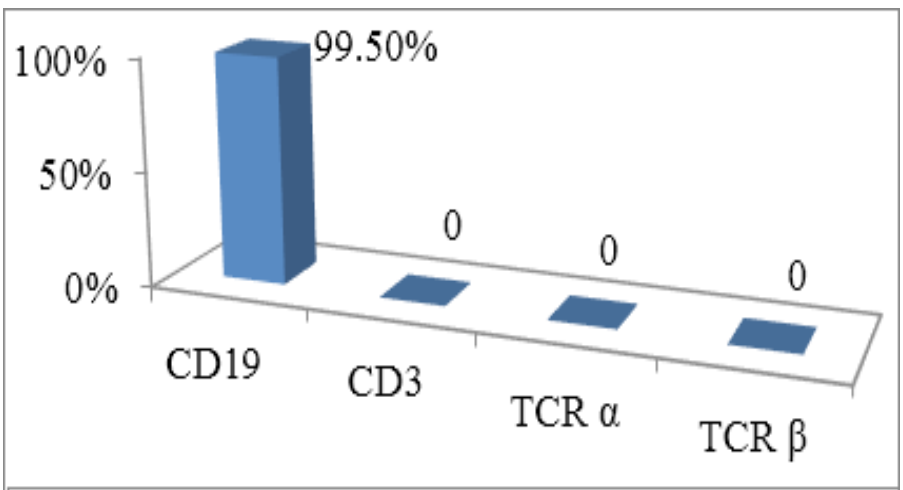

Figure 4: FACS analysis of enlarged lymph nodes. Almost all eGFP expressing cells were positive for CD19 and negative for CD3 TCR $a$ and TCR $\beta$.

\section{Tumour cells Control cells}

\section{$14.5 \mathrm{kDa}$}

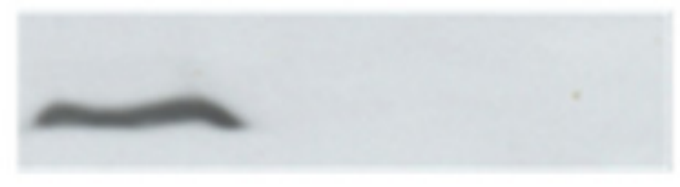

Figure 5: Western blot analysis. Tumor cells expressed p21SNFT protein $(14.5 \mathrm{kDa})$. Control cells did not express this protein.

\section{DISCUSSION}

In this study, we showed that retrovirus mediated transfer of $p 21 S N F T$ gene into polyclonal $\mathrm{T}$ lymphocytes and subsequent transplantation of these cells into mice induced lymphoid tumors of mature type, which resembled human peripheral $\mathrm{T}$ cell lymphoma. There are three central findings of our research work which support the causative role of p21SNFT in the development of lymphoma. First, all the tumor cells expressed the eGFP marker gene, which was confirmed by FACS analysis. Second, the tumor cells expressed p21SNFT gene on protein level, which was confirmed by performing Western blot analysis. Third, the tumors originated from the donor cell compartment, not from the recipient mice themselves, which was confirmed by using CD45.1 and CD45.2 markers. The donor cells were positive for CD45.1 and the recipients for CD45.2 marker. Hence, all eGFP expressing tumor cells were positive for CD45.1, which indicate that the tumors were induced in the p21SNFT transduced Tlymphocytes.

For further confirmation of the phenotype of tumor cells, we performed CD marker analysis, which revealed the loss of T cell markers (CD3 and TCR). Several studies have shown the loss of T cell markers and aberrant expression of CD markers by $\mathrm{T}$ cell lymphomas. A study on hepatosplenic T cell lymphoma showed that the tumor cells lost the expression of CD3. ${ }^{14}$ Another study also revealed loss of TCR marker in cutaneous $\mathrm{T}$ cell lymphoma. ${ }^{15}$ In our experiments, the tumor cells lost T cell markers and expressed CD19, which is a B cell marker. The aberrant expression of CD19 by T cell lymphoma cells was already described by several researchers. ${ }^{16-18}$

Another study reported a case of peripheral T-cell lymphoma, in which the cells not only expressed CD19, but also other B cell markers such as CD20 and CD79a. ${ }^{19}$

The tumors in our experimental mice developed after a longer latency period, which resemble some of the human lymphomas which develop after a longer latency period of years. ${ }^{20}$ The longer latency period indicates that in addition to the p21SNFT gene, there may have occurred other additional mutations which allowed the outgrowth of a transformed tumorigenic clone, resulting in the tumor mass with identical cell morphology. The longer latency period for the development of lymphoma have already been described. ${ }^{21}$ Hence, in this regard several cooperating genes may be involved in the tumorignesis in our experimental mice which need to be further explored by performing DNA microarray analysis. A recent study on genetic analysis of peripheral T cell lymphoma revealed expression profile of 155 genes which were differentially expressed in comparison to the normal T lymphocytes. Among those 155 genes, 64 were upregualted and 91 were downregulated. Majority of the upregulated genes were known to play a role in transcription, proliferation, cell adhesion and matrix remodeling, while many of the downregulated genes were known to play a role in apoptosis. All these events contribute to the development of lymphoma. ${ }^{22}$

In the current study, we used $\gamma$-retroviral vector to transfer p21SNFT into T lymphocytes. Although we used the control group of mice, transplanted with empty $\gamma$-retroviral vectors, from which none of the mice developed any malignancy, it might still be possible that $\mathrm{T}$ lymphocytes got transformed into malignant cells as a result of insertional mutagenesis by $\gamma$-retroviral vectors. Retroviral insertional mutagenesis, resulting in cancerous transformation of normal cells have already been previously reported. ${ }^{23,24}$ As the mature $\mathrm{T}$ lymphocytes could be transformed into malignant cells in our experimental set up, the safety issue of T lymphocytes in gene therapy should further be investigated.

Since we used limited number of mice in our experiments, further experiments on $211 S N F T$ with increased number of mice with more 
molecular analyses need to be carried out to better understand its pathogenic role in the development of lymphoma.

\section{CONCLUSION}

The results of our present study show that $\gamma$-retroviral mediated transfer of p21SNFT causes malignant transformation of polyclonal T lymphocytes.

\section{ACKNOWLEDGEMENT}

This study was funded by Deutsche Forschungsgemeinschaft, for which we are thankful to it.

\section{CONFLICT OF INTEREST}

The authors declare no conflict of interest.

\section{REFERENCES}

1. lacobelli M, Wachsman W, McGuire KL. Repression of IL-2 promoter activity by the novel basic leucine zipper p21SNFT protein. J Immunol. 2000;165(2):860-8.

2. Brune $V$, Tiacci $E$, Pfeil l, et al. Origin and pathogenesis of nodular lymphocytepredominant Hodgkin lymphoma as revealed by global gene expression analysis. J Exp Med. 2008;205(10):2251-68.

3. Schwering I, Bräuninger A, Distler $V$, et al. Profiling of Hodgkin's lymphoma cell line L1236 and germinal center B cells: Identification of Hodgkin's lymphomaspecific genes. Mol Med. 2003;9(3-4):85-95

4. Davis MM, Bjorkman PJ. T-cell antigen receptor genes and T-cell recognition. Nature. 1988;334(6181):395

5. Nikolich-Žugich J Slifka MK, Messaoudi I. The many important facets of T-cell repertoire diversity. Nat Rev Immunol. 2004;4(2):123.

6. Min B, Paul WE. Endogenous proliferation: Burst-like CD4 T cell proliferation in lymphopenic settings. Semin Immunol. 2005;17(3):201-7.

7. Troy $A E$, Shen $H$. Cutting edge: Homeostatic proliferation of peripheral $T$ lymphocytes is regulated by clonal competition. J Immunol. 2003;170(2):672-6

8. Clarke SR, Barnden M, Kurts C, Carbone FR, Miller JF, Heath WR. Characterization of the ovalbumin-specific TCR transgenic line OT-I: MHC elements for positive and negative selection. Immunol Cell Biol. 2000;78(2):110-7.

9. Mathas S, Hartmann S, Kueppers R. Hodgkin lymphoma: Pathology and biol- ogy. Semin Hematol. 2016;53(3):139-47.

10. Kadin ME, Drews R, Samel A, Gilchrist A, Kocher O. Hodgkin's lymphoma of Tcell type: Clonal association with a CD30+ cutaneous lymphoma. Hum Pathol. 2001;32(11):1269-72.

11. Bouard D, Alazard-Dany N, Cosset FL. Viral vectors: From virology to transgene expression. Br J Pharmacol. 2009;157(2):153-65.

12. Kumar. Transformation Potential of NPM-ALK, p21SNFT and Tax for Mature T Lymphocytes. PhD Thesis. University of Frankfurt, Germany. 2010.

13. Kumar A. Malignant transformation of mature $T$ cells after gammaretrovirus mediated transfer of nucleophosmin-anaplastic lymphoma kinase oncogene. Indian J Pathol Micr. 2015; 58(3): 301.

14. Chauhan R, Tyagi S, Mirgh S, et al. Expect the unexpected-Loss of surface CD3 on flow cytometry in hepatosplenic T-cell lymphoma: An eye opener. Indian J Pathol Micr. 2018;61(2):275.

15. Caballero A, Novelli S, Mozos A, Muret PG, Sierra J, Briones J. Silent T-cell receptor cutaneous T-cell lymphoma associated to a clonal plasma cell proliferation. Hematol Rep. 2019;11(1):7841.

16. Rizzo K, Stetler-Stevenson M, Wilson W, Yuan CM. Novel CD19 expression in a peripheral T cell lymphoma: A flow cytometry case report with morphologic correlation. Cytometry Part B: Clinical Cytometry: The Journal of the Internationa Society for Analytical Cytology. 2009;76(2):142-9.

17. Toyoda $Y$, Horikoshi $Y$, Tonouchi $T$, et al. CD5+, CD7+ and CD19+ non-Hodgkin's lymphoma in a child. The Japanese Journal of Clinical Hematology. 1991;32(2):137-41.

18. Jain $H$, Shetty $D$, Jain $H$, Sengar $M$, Khattry $N$, Subramanian P. A rare case of hepatosplenic $\gamma \delta$ T-cell lymphoma expressing CD19 with ring chromosome 7 and trisomy 8. Cancer Genet. 2018;228:17-20.

19. Yao X, Teruya-Feldstein J, Raffeld M, Sorbara L, Jaffe ES. Peripheral T-cell lymphoma with aberrant expression of CD79a and CD20: A diagnostic pitfall. Modern Pathol. 2001;14(2):105.

20. Racke F, Simpson S, Christian B, Blum KA, Hasserjian R, Zhao W. Evidence of long latency periods prior to development of mantle cell lymphoma. Am Soc Hematology. 2010;116(21):323.

21. Kuefer MU, Look AT, Pulford K, et al. Retrovirus-mediated gene transfer of NPM-ALK causes lymphoid malignancy in mice. Blood. 1997;90(8):2901-10.

22. Piccaluga PP, Agostinelli C, Califano A, et al. Gene expression analysis of peripheral T cell lymphoma, unspecified, reveals distinct profiles and new potential therapeutic targets. J Clin Invest. 2007;117(3):823-34.

23. Davé UP, Jenkins NA, Copeland NG. Gene therapy insertional mutagenesis insights. Science. 2004;303(5656):333.

24. Uren A, Kool J, Berns A, Lohuizen MV. Retroviral insertional mutagenesis: Past, present and future. Oncogene. 2005;24(52):7656-72.

Article History: Submission Date :03-02-2020 ; Revised Date : 15-02-2020; Acceptance Date : 17-03-2020.

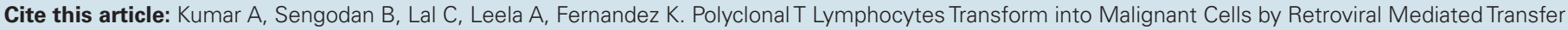
of p21SNFT Gene. J Young Pharm. 2020;12(1):71-4. 